\title{
Os limites da ação coletiva nos Conselhos Municipais de Saúde
}

The limits of collective action in municipal health councils

Milton Cordeiro Farias Filho ${ }^{1}$

Andréia Neves da Silva ${ }^{2}$

Armin Mathis ${ }^{2}$

Superintendência de Pesquisa, Universidade da Amazônia. Av. Alcindo Cacela 287, Umarizal. 66.060-902 Belém PA Brasil.

mcffarias@gmail.com

${ }^{2}$ Núcleo de Altos Estudos

Amazônicos, Universidade Federal do Pará.
Abstract This article addresses the collective actions of participation in the municipal health councils. The underlying research sought to establish the performance of the councilors in the municipal health councils in the Metropolitan Region of Belém in the State of Pará. A survey of documents for the 2005-2010 period, structured interviews and observations with counselors in sessions of meetings of the boards of three counties were analyzed. An indicator of three levels of participation centered on the legal functions of the health councils and based on the theory of social groups as its analytical principle was constructed. The results indicate that the activities of the councils are marked by the co-option of councilors and the definition of deliberative agendas on the part of representatives of management, in which individual interests predominate over the collective interests of the councilors in the deliberations. The conclusion is that such behavior is contrary to the principles of the Unified Health System - SUS and weakens the social image of the councils, in addition to opening up a new analytical perspective.

Key words Councils, Collective Action, Individual Interests, Participation
Resumo O artigo traz uma abordagem sobre as ações coletivas de participação nos conselhos municipais de saúde. A pesquisa que o fundamenta teve como objetivo verificar a atuação dos conseIheiros nos conselhos municipais de saúde da Região Metropolitana de Belém (PA). Foi utilizado levantamento documental (período 2005 a 2010), entrevistas estruturadas com conselheiros e observações em sessões de reuniões dos conselhos de três municípios. Foi construído um indicador de três níveis de participação com base nas funções legais dos conselhos de saúde, tendo como fundamento analítico a teoria dos grupos sociais. Os resultados indicam que as ações dos conselhos são marcadas por cooptação de conselheiros, definição de agendas deliberativas por parte de representantes da gestão, predominando os interesses individuais dos conselheiros sobre os interesses coletivos nas deliberações. Concluiu que tais comportamentos contrariam os princípios do Sistema Único de Saúde - SUS e fragiliza a imagem social dos conselhos, além de abrir uma nova perspectiva de análise.

Palavras-chave Conselhos, Ação coletiva, Interesses individuais, Participação 


\section{Introdução}

A Constituição Federal (CF) de 1988 direcionou diversas políticas para a gestão das esferas estadual e municipal de governo, fato este referenciado pela literatura como descentralização de políticas públicas ${ }^{1}$. Neste aspecto, deu-se destaque à participação da sociedade como uma forma de controle social da gestão pública². O Sistema Único de Saúde (SUS), resultado do Movimento de Reforma Sanitária dos anos 1980, a criação do Conselho Nacional de Saúde (CNS) e as resoluções específicas (33/1992 e 333/2003) para a área, assim como os atos executivos como as Normas Operacionais Básicas (NOB $)^{3,4}$ são aspectos relevantes na área de saúde pública.

A regulamentação para a área de saúde pública vem da Lei 8.142/1990 selhos e conferências de saúde; da Lei 8080/1990 (Lei Orgânica da Saúde) e Lei 8074/19907 (Lei de Diretrizes Orçamentárias) que auxiliam a organização do sistema como um todo. Assim, a partir da regulamentação do conselho de saúde e de seu funcionamento, a literatura sobre o tema se amplia, apresentando estudos de variados enfoques.

A evidência do interesse dos pesquisadores pelo tema se mostrou nos estudos que tratam do conselho de saúde enquanto instância de participação social para controle social da gestão e da política de saúde nos municípios. Em recente revisão da literatura ${ }^{8}$, foi constatada uma ênfase na participação da sociedade, em termos históricos, com destaque para os fundamentos do conselho como forma de controle social. Basicamente, os eixos temáticos desses estudos são o papel do Estado, na democracia participativa e comunitária promovida pela institucionalização do conselho, promoção da saúde, organização social e análise do arcabouço institucional.

Os trabalhos que sustentam a participação nos conselhos de saúde como fundamento da cidadania defendem que eles geram um aprendizado que forma sujeitos críticos ${ }^{9}$, mas evidenciam seus limites quanto a participação efetiva dos conselheiros, seja por condições desiguais entre os participantes, refletida na assimetria da composição dos conselhos e pelas dificuldades impostas pela cultura política ${ }^{10}$, ou pela superposição dos interesses da gestão municipal, que exerce controle sobre a agenda dos conselhos de saú$\mathrm{de}^{11}$. Essas dificuldades destacadas nos estudos sobre os conselhos municipais de saúde se fundamentam também na complexidade e abrangência das atribuições dos conselhos, que impõe limites à atuação plena, já que no interior dos conselhos há uma reprodução de certos vícios da sociedade e de suas práticas políticas ${ }^{12,13}$.

Estas abordagens demonstram que os conselhos apenas reproduzem comportamentos da diferenciação socioeconômica que por sua vez impõem limites à participação social na gestão pública, que se reflete na estrutura e funcionamento dos conselhos e que, de certa forma, compromete a legitimidade social dessa instância deliberativa.

Outras perspectivas de análise nas pesquisas partem, na maioria dos casos, da atuação dos conselhos, tendo como foco os conselheiros usuários e sustentam que estes apresentam comportamentos similares. Esses trabalhos demonstram que os conselheiros usuários privilegiam as motivações pessoais e os interesses individuais e que estes se manifestam na troca de favores e favoritismo no uso dos serviços, desvirtuando a função representativa e comprometendo a visibilidade social dos conselhos municipais de saúde, enquanto espaço de deliberação para o controle social da gestão ${ }^{13-15}$. Assim, os conselhos, devido sua baixa capacidade gerencial, têm dificuldade de fazer com que a gestão municipal consiga cumprir os princípios do Sistema Único de Saúde - SUS ${ }^{16}$.

Diante desse panorama, a pesquisa partiu das seguintes questões: De que forma ocorre a participação nos conselhos municipais de saúde em municípios da Região Metropolitana de Belém? De que forma se dá o comportamento participativo dos conselheiros destes conselhos municipais?

A premissa teórica que sustenta essas questões é que a participação dos conselheiros está baseada em quatro tipos de comportamento dos agentes (membros dos conselhos): a) agir de forma individual para obter benefícios individuais; b) agir de forma individual para alcançar benefícios coletivos; d) agir de forma coletiva para almejar benefícios individuais; e) agir de forma coletiva e buscar benefícios coletivos.

Para teoria dos grupos sociais ${ }^{17}$, quando os indivíduos de um grupo conduzem suas ações de forma racional, mesmo que consigam alcançar os objetivos grupais (coletivos), sempre agirão voluntariamente buscando seus interesses individuais. A questão central da teoria é: O que leva os indivíduos se associarem e se manterem associados? Do ponto de vista da racionalidade coletiva, todos ganhariam caso houvesse cooperação de todos. No entanto, isso não acontece sempre, o que leva indivíduos a agirem racionalmente medindo os custos e benefícios de suas ações coletivas em organizações ou grupos. Logo, forma-se uma relação contraditória entre uma racionalidade individual e racionalidade coletiva ${ }^{17}$. 
A premissa da teoria sustenta que o ator racional, quando se tratar de um bem público, se orienta pela lógica individual e não coletiva. Isso aumenta a complexidade da regulação e do papel do governo na gestão social, assim como promove a organização de grupos de interesse no interior da sociedade, na condução de determinadas políticas ${ }^{17}$.

As organizações de ação coletiva reduzem a eficiência e os ganhos da sociedade que representam, porque seus membros estão sempre em conflito entre interesses individuais e coletivos. Os membros, de forma individual, buscarão sempre receber benefícios advindos dos esforços coletivos, aumentando assim a parcela de benefício total gratuitamente ${ }^{17}$. Na lógica da ação coletiva, da teoria, o conjunto de indivíduos com interesses comuns tem dificuldades de se organizar porque o elevado custo da participação para a busca do bem coletivo favorece o comportamento individual do tipo "carona" (free rider). Dessa forma um indivíduo se beneficia dos ganhos auferidos pelo grupo (benefício coletivo) sem arcar com os custos individuais para isso ${ }^{17}$.

Outro fator de destaque na teoria é a grande assimetria entre os membros de um grupo, medida a partir dos recursos disponíveis e níveis de interesses por um benefício coletivo. Esta assimetria pode gerar a exploração entre membros, quando um membro ou uma parte deles assume os maiores custos para o provimento do bem público, reduzindo assim seus benefícios individuais ${ }^{17}$.

Com base nos aportes teóricos, a pesquisa teve como objetivo verificar as formas de participação nos conselhos e o comportamento dos conselheiros nas funções dos conselhos municipais de saúde em municípios da Região Metropolitana de Belém (PA).

A pesquisa buscou apresentar uma contribuição para literatura que trata da atuação dos conselhos municipais de saúde, em função do crescente interesse pelo tema. Este fato justifica esta abordagem e o uso da teoria dos grupos sociais como fundamento analítico para verificar comportamentos de participação nos conselhos e indicar em que medida está havendo uma busca por benefícios coletivos (para os usuários da saúde pública) por bens públicos (os serviços ofertados pelo SUS)

\section{Método}

A pesquisa ocorreu em três dos cinco municípios da Região Metropolitana de Belém (PA): Belém, Marituba e Benevides. Os outros municípios da região foram excluídos da pesquisa por falta de acesso a informações completas. Os procedimentos adotados foram:

(a) Levantamento documental ${ }^{18}$, cujas fontes foram as atas de reunião dos conselhos, regimentos internos, legislação de criação dos conselhos nos municípios, documentos com as deliberações das reuniões e relatórios de gestão das secretarias de saúde (2005 a 2010).

(b) Observações sistemáticas ${ }^{19}$ em reuniões ordinárias dos conselhos (oito sessões com tempo variando de acordo com a duração de cada reunião) e nas instalações das secretarias de saúde.

(c) Entrevistas estruturadas ${ }^{18}$, usando um roteiro que foi organizado em seis dimensões (relações governo e sociedade civil; disseminação das informações; processo de decisão nos conselhos; perfil dos conselheiros; fiscalização dos serviços; ações de saúde). Os critérios para seleção dos entrevistados foram: i) ser membro titular do conselho; ii) ter mais de um mandato como conselheiro; iii) concordância em participar da pesquisa como entrevistado (de acordo com os critérios da Resolução $\left.n^{\circ} 196 / 96^{20}\right)$. Os mandatos cobertos pela pesquisa foram 2005-2006, 2007-2008 e 2009-2010.

A gestão 2010-2012 não foi objeto de pesquisa porque ainda estava em andamento e não havia informações suficientes de suas ações. No entanto, esta gestão serviu de referência para outras análises, pois as observações foram realizadas durante a mesma. O Quadro 1 mostra a distribuição das entrevistas por município e segmento representado nos conselhos.

Para verificação da participação nos conselhos, foi desenvolvida uma escala de valores (que variou entre 0,00 e 3,00) para as funções de participação do conselho. A partir da escala foram criados três níveis de atuação (indicador de frequência), são eles:

Nível 1: o conselho não cumpre plenamente sua função. Foi aplicado quando as informações indicaram que não houve interferência e/ou controle do conselho nas ações de saúde do município. Nesse caso o conselho não cumpriu seu papel e recebeu nota de 0,00 a 1,00. Quando este fato se revelou, houve manifestação de ação individual para busca de benefícios individuais ou de ação coletiva para busca de benefícios individuais por parte dos conselheiros. 


\begin{tabular}{|c|c|c|c|}
\hline \multicolumn{4}{|l|}{914} \\
\hline \multirow[t]{5}{*}{ 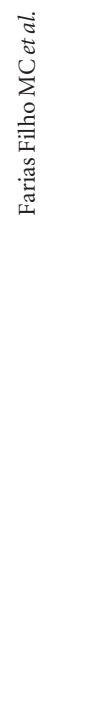 } & Quadro 1. Número & vistas por município & uisado. \\
\hline & Município & Conselheiros & $\mathrm{N}^{\circ}$ de entrevistas por segmento \\
\hline & Belém & $\begin{array}{l}20 \text { conselheiros } \\
\text { (4 entrevistados) }\end{array}$ & $\begin{array}{l}2 \text { Usuários } \\
1 \text { Profissional de saúde } \\
1 \text { Gestor/Prestador de serviço }\end{array}$ \\
\hline & Marituba & $\begin{array}{l}12 \text { conselheiros } \\
\text { (4 entrevistados) }\end{array}$ & $\begin{array}{l}2 \text { Usuários } \\
1 \text { Profissional de saúde } \\
1 \text { Gestor/Prestador de serviço }\end{array}$ \\
\hline & Benevides & $\begin{array}{l}12 \text { conselheiros } \\
\text { (*12 entrevistados) }\end{array}$ & $\begin{array}{l}6 \text { Usuários } \\
3 \text { Profissional de saúde } \\
3 \text { Gestor/Prestador de serviço }\end{array}$ \\
\hline
\end{tabular}

"Os 12 conselheiros foram entrevistados porque o município foi o teste e todas as informações foram aproveitadas para análises.

Nível 2: o conselho exerce suas ações, mas de maneira instrumental/formal. Foi aplicado quando as informações mostraram ações em várias direções, superficiais, apenas para mostrar que se cumpriu o que regem as normas formais e/ou quando ações foram discutidas nas reuniões do conselho, mas sem o caráter deliberativo, ocorrendo situação semelhante a denominada de "conselho burocrático"21. Nessa situação o conselho cumpriu parcialmente seu papel e recebeu nota de 1,01 a 2,00. Quando este resultado surgiu houve demonstração de ação coletiva para almejar benefícios individuais por parte dos conselheiros.

Nível 3: a participação foi considerada efetiva. Foi aplicado quando os resultados da pesquisa indicaram que o conselho realmente participou de forma efetiva nas ações de saúde do município. Nesse caso o conselho cumpriu integralmente seu papel e recebeu nota de 2,01 a 3,00. Logo, houve manifestação de ação individual para obter benefícios coletivos ou ação coletiva para buscar resultados coletivos por parte dos conselheiros.

O Quadro 2 mostra a classificação dos níveis de participação usada na pesquisa e as notas atribuídas resultantes da fórmula a seguir:

$$
\mathbf{N}=\frac{\text { Vn (ano) }}{\text { nSIF }}
$$

$\mathbf{N}=$ Nível de Participação.

Vn = Valor atribuído a cada subitem da Função nSIF = número de Subitens da Função.

Para a análise dos resultados foram adotados os seguintes procedimentos:

a) O conteúdo das entrevistas e dos documentos passou pela técnica de análise de conteúdo. Para isso foi construída uma matriz de análise ${ }^{18}$. b) Os dados (Tabela 1) foram organizados em planilhas para construção da escala de pontuação, utilizando-se as médias de verificação dos níveis de participação dos conselheiros, para avaliação e comparação final com as informações resultantes das entrevistas e dos documentos.

\section{Resultados e Discussão}

Os resultados dos níveis de participação dos conselhos de saúde dos municípios estudados (Quadro 2) mostram que na função 1 (controle de gastos), relacionada com a política e o planejamento da área de saúde, indicam a predominância do nível de participação instrumental/formal em todos os municípios, durante o período estudado, já que foi baixa a frequência dos níveis de participação $(1,01$ e 2,00).

No município de Marituba, nas duas primeiras gestões, o conselho alcançou o nível de participação efetiva, destacando-se dos demais. No entanto, no primeiro ano da gestão 2009-2010 houve redução no nível de participação, configurando uma atuação do tipo instrumental/formal, mesmo considerando a ausência de dados do ano de 2010.

O conselho com o pior desempenho foi o de Benevides, que não alcançou o nível de participação efetiva $(2,01$ a 3,00). Na função 1 (controle dos gastos) neste conselho, nas duas primeiras gestões, predominou o nível sem participação $(0,00$ a 1,00$)$ e participação instrumental/formal $(1,01$ a 2,00$)$, o que demonstra que os interesses individuais prevaleceu sobre os interesses coletivos, tal qual previsto na teoria ${ }^{17}$, por ausência de participação efetiva dos conselhos na busca de melhoria do acesso por bens públicos. Já os con- 


\begin{tabular}{|c|c|}
\hline Funções do Conselho Municipal de Saúde (CMS) $)^{18-20}$ & $\begin{array}{c}\text { Níveis das } \\
\text { Funções do CMS }\end{array}$ \\
\hline $\begin{array}{l}\text { F1: Controle dos gastos: } \\
\text { 1.1. Análise do Plano Plurianual (PPA) da Secretaria Municipal de Saúde; } 1.2 \text {. } \\
\text { Avaliação e acompanhamento dos critérios de movimentação de recursos repassados à } \\
\text { Secretaria Municipal de Saúde; 1.3. Análise da prestação de contas trimestral da } \\
\text { Secretaria Municipal de Saúde; 1.4. Avaliação do Relatório de gestão; }\end{array}$ & \multirow{4}{*}{$\begin{array}{c}\text { Sem } \\
\text { participação } \\
(0,00 \text { a } 1,00) \\
\\
\text { Participação } \\
\text { instrumental/ } \\
\text { formal } \\
(1,01 \text { a } 2,00) \\
\text { Participação } \\
\text { efetiva } \\
(2,01 \text { a } 3,00)\end{array}$} \\
\hline $\begin{array}{l}\text { F2: Deliberação das ações: } \\
\text { 2.1. Avaliação do funcionamento dos serviços de saúde; } 2.2 \text {. Acompanhamento das } \\
\text { ações e serviços de saúde no município; } 2.3 \text {. Avaliação das reuniões anteriores do CS } \\
\text { para averiguar a efetivação das deliberações feitas à rede de atendimento do Sistema } \\
\text { Municipal de Saúde; } 2.4 \text {. Visitas regulares nos estabelecimentos de saúde, para } \\
\text { fiscalizar seu funcionamento. }\end{array}$ & \\
\hline $\begin{array}{l}\text { F3: Denúncias de irregularidades: } \\
\text { 3.1. Criação de Câmaras e comissões de fiscalização, para averiguação das denúncias; } \\
\text { 3.2. Discussão nas reuniões para examinar as denúncias e as irregularidades nos } \\
\text { serviços; 3.3. Apresentação de relatório dos casos nas reuniões; } 3.4 \text {. Apresentação de } \\
\text { parecer do CS sobre a irregularidade denunciada; } 3.5 \text {. Encaminhamento das } \\
\text { denúncias para os órgãos responsáveis. }\end{array}$ & \\
\hline $\begin{array}{l}\text { F4: Divulgação das ações do CS: } \\
\text { 4.1. Informação à comunidade da agenda de reuniões (Data e local) do CS; } 4.2 \text {. } \\
\text { Informação das funções e competências do CS nos meios de comunicação local } \\
\text { (jornal, revista, rádio etc.); 4.3. Apresentação de relatório de atividades do CS; } 4.4 \text {. } \\
\text { Formulação de Panfletos, cartilhas produzidas pelo CS; 4.5. Realização de audiências } \\
\text { públicas. }\end{array}$ & \\
\hline
\end{tabular}

* Informações baseadas nos regimentos dos conselhos, legislação de criação e atas de reunião e outras informações coletadas na pesquisa.

selhos de Belém e Marituba, durante todo o período, manifestaram o tipo de participação instrumental/formal e efetiva, o que sustenta a premissa teórica de busca por benefícios individuais em ações coletivas.

Na função 2 (deliberação das ações) os resultados indicam que o conselho de Benevides teve o pior desempenho entre os conselhos pesquisados, com predominância do nível "sem participação" $(0,00$ a 1,00$)$ em todas as funções. Já os conselhos de Belém e de Marituba apresentaram níveis de participação mais elevados, pois alcançaram o nível de participação efetiva $(2,01$ a 3,00).

$\mathrm{Na}$ função 3 (denúncias de irregularidades), o conselho de Belém obteve o nível de participação efetiva. Porém, no último ano do período estudado o desempenho diminuiu para o pior nível (sem participação 0,00 a 1,00). Este fato se refletiu na intensa divulgação na imprensa local de irregularidades no uso de verbas da saúde.

O conselho do município de Marituba, apenas no ano de 2009, apresentou um nível de par- ticipação efetiva na função 3 . Nos demais anos o nível foi instrumental/formal. Já o conselho do município de Benevides apresentou uma leve melhora nos níveis de participação na última gestão estudada, mantendo-se no nível de participação instrumental/formal. Neste município, nas duas primeiras gestões do conselho (últimos anos) foram marcados pela ausência de participação (sem participação 0,00 a 1,00 ).

$\mathrm{Na}$ função 4 (divulgação das ações do conselho), os conselhos se comportaram de forma semelhante. No conselho de Belém os níveis oscilaram entre sem participação $(0,00$ a 1,00$)$ e participação instrumental/formal $(1,01$ a 2,00$)$ e o de Marituba se comportou da forma semelhante, mesmo sem informações do último ano do período estudado. O conselho de Benevides se manteve todo o período com o nível mínimo (sem participação 0,00 a 1,00).

Os resultados conjuntos (Tabela 1) expressam que os conselhos de saúde nos municípios, ainda que sejam uma instância de participação 


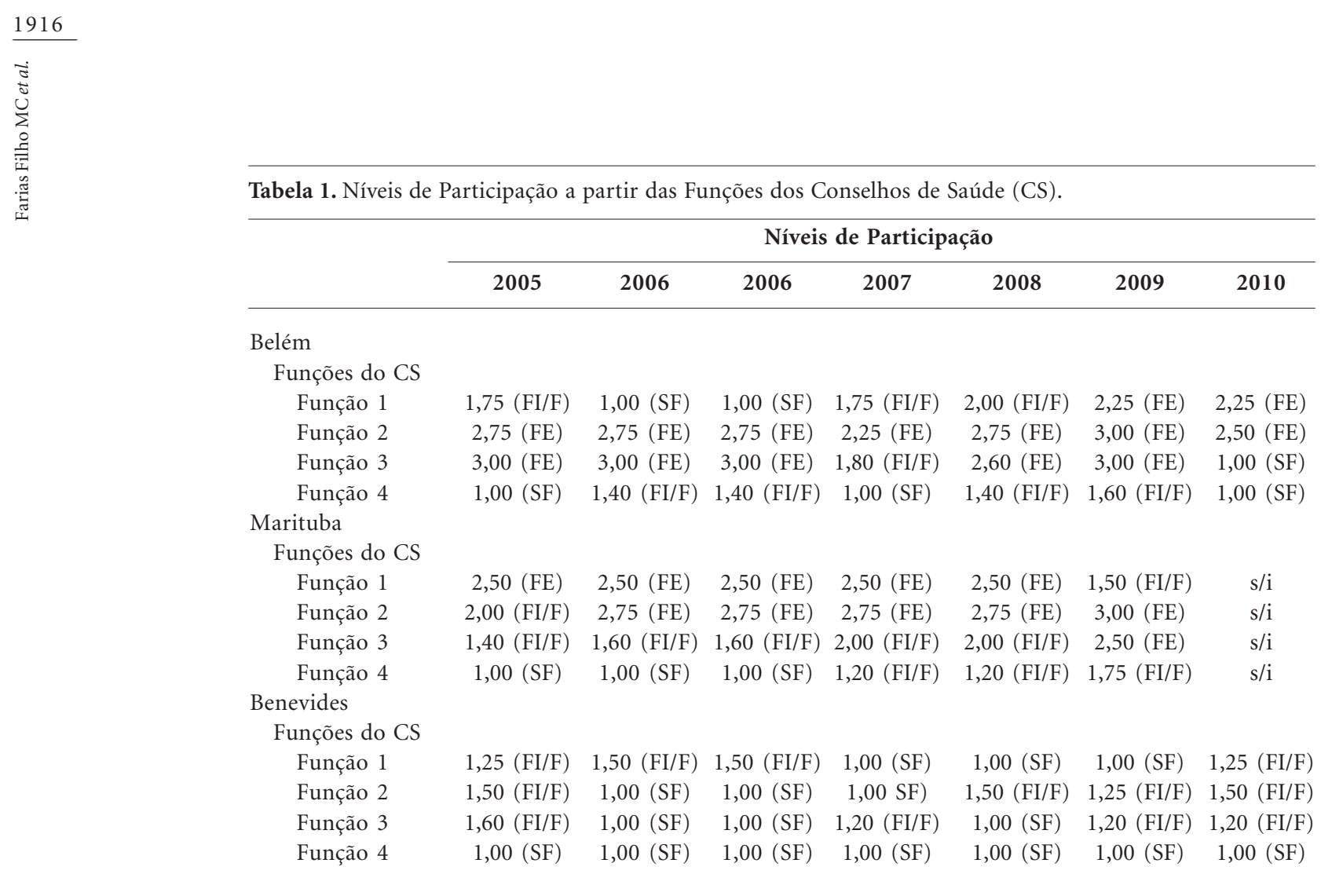

Fonte: Elaboração própria, a partir dos dados e informações coletados e comparados.

$\mathrm{SF}=$ Sem Participação = 0,00 a 1,00; FI/F = Participação Instrumental/Formal = 1,01 a 2,00; FE = Participação Efetiva = 2,01 a 3,00

social e de caráter deliberativo, exercem pouco o controle social (da sociedade para o Estado), cujos limites de atuação foram constatados em outras realidades nacionais ${ }^{9,12,13,15}$, o que reflete desvios dos princípios de sua existência ${ }^{6,22}$ e que pode ter relação com a dificuldade de exercício de suas atribuições ${ }^{11}$, em razão dos poucos conhecimentos técnicos e elevados custos de oportunidade de participação ${ }^{14,17}$ por parte dos conselheiros usuários.

De modo geral os resultados das entrevistas com conselheiros evidenciam as dificuldades de participação nos conselhos, no que se refere ao controle social efetivo. Dentre os principais fatores encontrados para este comportamento merecem destaque:

a) os conselheiros têm dificuldades em compreender seu papel no conselho. Alguns entrevistados (conselheiros) perceberam que, nos termos da teoria ${ }^{17}$, os custos individuais de participação são maiores que seus benefícios e estes são reduzidos e concentrados em poucos segmentos sociais.

[...] Tem que melhorar, por que acontece o seguinte: ás vezes o conselheiro está lá e não sabe nem por que ele é conselheiro [...] (Entrevistado 19).
[...] Eu acho que a maior dificuldade é, a questão do custeio, eu acho que os conselheiros têm a dificuldade de se locomover, o conselho não tem uma infraestrutura boa, para fazer a fiscalização, temos que solicitar carros, e há uma dificuldade em disponibilizar carro [...] (Entrevistado 9).

[...] não tem uma ajuda de custo para locomoção, um conselheiro tem que pegar um ônibus para ir lá na unidade fiscalizar [...] (Entrevistado 6).

[...] As principais dificuldades do conselheiro atuar é ter tempo. Você tem várias atividades, a pessoa trabalha. A questão de dedicar um tempo maior é uma dificuldade [...] (Entrevistado 17).

Os relatos mostram evidências de baixa capacidade de atuação efetiva dos conselhos, quando se compara com suas funções. No entanto, a tese de postura de submissão dos conselheiros usuários ${ }^{10}$ encontra limites diante dos resultados encontrados, pois os trechos das entrevistas demonstram que as dificuldades dos conselheiros usuários estão mais na compreensão de seu papel e de fazer uso do poder político representativo que têm na função (atuação proposicional e fiscalizadora), do que na postura de submissão. Este comportamento desvirtua a atuação, pois o que se espera é que predomine a busca por interesses coletivos (para a sociedade) e 
não em busca por benefícios individuais ${ }^{14,17}$, como evidenciam as situações de troca de favores na atividade de representação.

[...] aquelas pessoas que querem trabalhar pela comunidade, é uma minoria bem restrita, pelo menos eu vejo nesse conselho, que usa isso em vantagem própria [...] (Entrevistado 8).

[...] se tu precisa entrar com uma pessoa numa unidade de saúde com urgência, e não tem médico; aí quando se identifica como conselheiro a história muda, já aparece um médico ali para te aten$\operatorname{der}[\ldots]$ (Entrevistado 17).

[...] alguns estão lá para cumprir acordos, está lá para fazer uma relação com o governo [...] às vezes o conselheiro está lá e não sabe nem por que ele é conselheiro, ele está para representar um grupo e uns interesses por trás de algumas coisas, então esquece de defender seu grupo de saúde [...] (Entrevistado 4).

b) Mesmo encontrando comportamentos evidentes da busca por interesses individuais, o reduzido papel dos conselheiros usuários também é resultado da concentração de poder nos representantes da gestão municipal ${ }^{11,12}$. Além disso, os conselhos têm pouca visibilidade social ${ }^{13}$ porque seu funcionamento, de acordo com a legislação ${ }^{22}$, o faz uma instância mais deliberati$\mathrm{va}^{12,16}$, porém o processo deliberativo encontra limites na dinâmica de composição assimétrica do poder nos conselhos pesquisados.

[...] ou o conselheiro se dobra à gestão e aprova tudo que ela quer, ou ele se torna objeção à gestão, e não vai demorar muito no conselho, porque a sua própria entidade de origem, articulações políticas do município, tem forças que operam para tirar fulano do conselho [...] (Entrevistado 5).

[...] tem que padronizar na verdade as eleições dos conselhos municipais, por que eu acho muito aberto, muito frágil, a forma do sistema eleitoral nos conselhos de saúde, há uma cooptação dos governos em cima dos conselheiros [...] (Entrevistado 16).

[...] o conselho não tem autoridade pra ele dizer assim: 'mostra aí a relação do pessoal que fez ultrassonografia pra ver se de fato não tem mais' [...] (Entrevistado 12).

Os resultados demonstram que os conselhos, uma importante instância de participação social, ainda tem muito que avançar. O elevado custo individual para os membros dos conselhos se manifesta em cooptação de conselheiros usuários, baixa frequência nas sessões do conselho, poucas ações de caráter deliberativo, oportunismo de conselheiros usuários, entre outros comportamentos, demostram que a busca por bene- fícios individuais supera a busca por benefícios coletivos, na forma como sustenta a teoria dos grupos sociais da ação social ${ }^{17}$.

Também ficou evidente que a ação coletiva para busca de interesses individuais e a ação individual em busca de interesses também individual foi o que prevaleceu como comportamento dos conselheiros usuários pesquisados. Os demais conselheiros (representante da outras categorias na representação paritária) também manifestaram esse comportamento, porém não demonstram claramente as motivações, já que o discurso do coletivo está presente em todos os conselheiros.

Mesmo que involuntariamente, percebeu-se que o comportamento de busca por interesses coletivos predominou no interior dos conselhos. Nos termos da teoria, a questão é saber: o que leva os indivíduos se manterem associados (ou no conselho)? Os resultados encontrados mostram que os interesses são diversos. Do lado dos representantes do governo, busca-se manter o controle sobre os conselheiros (controle de agenda de deliberação) como forma de conduzir a gestão programada. Do lado dos conselheiros usuários, são diversas as motivações, indo desde fortalecimento político da comunidade, busca por privilégios no uso dos serviços e negociação de outros favorecimentos pessoais.

As pesquisas que sustentam a literatura sobre conselhos de saúde e que tratam do comportamento dos conselheiros não evidenciam os custos da participação para os usuários. Neste caso, a teoria mostra que a busca por bens coletivos tem incentivos coletivos e individuais, pois a participação tem um custo individual (custo de oportunidade), por isso novos estudos podem se voltar para as dificuldades dessa forma de participação, do ponto de vista dos custos individuais efetivos para cada categoria de conselheiro.

\section{Conclusão}

Os argumentos de parte da literatura que sustentam a presença de fenômenos como assimetria na composição dos conselhos gestores de saúde, controle de agenda deliberativa por parte dos representantes do governo e comportamento de submissão por parte de conselheiros usuários, não são capazes de construir um modelo generalizante de comportamento esperado de participação efetiva nos conselhos municipais de saúde.

É fato que a cooptação de conselheiros usuários existe e faz parte do jogo político em instân- 
cias deliberativas (como os conselhos). Entretanto, a ação coletiva nessas instâncias tem como princípio a busca de interesses coletivos do bem público em questão. Porém, o que se revelou foi a sobreposição dos interesses individuais em relação aos coletivos, comprometendo a apropriação pelo coletivo do bem público.

A cooptação de conselheiros é uma estratégia que os representantes do governo utilizam para obter o controle da agenda deliberativa nos conselhos e é um comportamento político comum em instâncias deliberativas. Da mesma forma que revela que os cooptados têm um benefício individual esperado ao adotarem tal comportamento, o que sustenta a tese da teoria dos grupos sociais de que os custos da participação, por serem elevados, favorecem a busca por interesses individuais em contexto de escolha por bens públicos, neste caso a saúde pública universal é um bem público e o conselho um grupo social para decisões coletivas que busca benefícios coletivos para uso deste bem público.

Parte da literatura sustenta que as ações deliberativas dos conselhos são limitadas porque ainda prevalecem características da cultura política de pouca participação e de submissão, porém é importante considerar também que outras características comprometem o resultado social dos conselhos, enquanto instância de decisão colegia- da, são elas: a) a estrutura organizacional e funcional dos conselhos não previne a cooptação; b) a sobreposição dos interesses individuais é difícil de ser combatida; c) a participação social como forma de controle da gestão da saúde pública municipal é mais complexa do que um aprendizado político ou forma de democracia participativa; d) a maioria dos problemas identificados nos conselhos foi em pesquisas realizadas em regiões metropolitanas, logo é possível que em pequenos municípios, distantes dos grandes centros urbanos, os problemas devam ser mais graves, o que indica que novas pesquisas em municípios distantes dessas regiões podem revelar novidades.

Novas pesquisas podem ser desenvolvidas para verificar a imagem institucional dos conselhos de saúde perante a sociedade, especialmente focando na percepção de sua efetividade como instância de gestão participativa junto aos usuários dos serviços de saúde, podem também revelar outros aspectos que não foram identificados com esta pesquisa, especialmente quando este olhar se volta para a percepção do usuário sobre o papel dos conselhos. Afinal é para eles que o sistema foi construído (SUS) e eles devem ter uma percepção de seus representantes (conselheiros) nesta instância deliberativa que auxilia na gestão desse bem público.

\section{Colaboradores}

MC Farias Filho, NA da Silva, e A Mathis participaram igualmente da concepção, delineamento, análise e interpretação dos dados, redação e revisão crítica e aprovação da versão final do artigo. 


\section{Referências}

1. Agustini J, Nogueira VMR, A descentralização da política nacional de saúde nos sistemas municipais na linha da fronteira Mercosul. Serv Soc Soc 2010; 102:222-243.

2. Oliveira VC. Comunicação, informação e participação popular nos conselhos de saúde. Saúde Soc 2004; 13(2):56-69.

3. Côrtes SV. Sistema único de saúde: espaços decisórios e a arena política de saúde. Cad Saude Publica 2009; 25(7):1626-1633

4. Batista AA, Muniz JN, Ferreira Neto JA, Cotta RMM. A contribuição da pesquisa de avaliação para o processo de implementação do controle social no SUS. Saúde Soc 2010; 19(4):784-793.

5. Brasil. Lei 8.142 , de 28 de dezembro de 1990. Dispõe sobre a participação da comunidade na gestão do Sistema Único de Saúde (SUS) e sobre as transferências intergovernamentais de recursos financeiros na área da saúde e dá outras providências. Diário Oficial da União 1990; $31 \mathrm{dez}$.

6. Brasil. Lei 8.080 , de 19 de setembro de 1990. Dispõe sobre as condições para a promoção, proteção e recuperação da saúde, a organização e o funcionamento dos serviços correspondentes e dá outras providências. Diário Oficial da União 1990; 19 set.

7. Brasil. Lei 8074 , de 31 de julho de 1990. Dispõe sobre as diretrizes orçamentárias para o ano de 1991 e dá outras providências. Diário Oficial da União 1990; 1 ago.

8. Cavalcanti MLT, Cabral MHP, Antunes LR. Participação em saúde: uma sistematização de artigos publicados em periódicos brasileiros - 1988/2005. Cien Saude Colet 2012; 17(7):1813-1823

9. Santos SF, Vargas AMD, Lucas SD. Conselheiros usuários do conselho municipal de saúde de Belo Horizonte: características sociais e representatividade. Saúde Soc 2011; 20(2):483-495.

10. Oliveira LC, Pinheiro R. A participação nos conselhos de saúde e sua interface com a cultura política. Cien Saude Colet 2010; 15(5):2455-2464

11. Bispo Júnior JP, Gerschman S. Potencial participativo e função deliberativa: um debate sobre a ampliação da democracia por meio dos conselhos de saúde. Cien Saude Colet 2013; 18(1):7-16.

12. Zambon VD, Ogata MN. Configurações dos conselhos municipais de saúde de uma região no estado de São Paulo. Rev Esc Enferm USP 2011; 45(4):890-
13. Vieira M, Calvo MCM. Avaliação das condições de atuação de conselhos municipais de saúde no estado de Santa Catarina, Brasil. Cad Saude Publica 2011; 27(2):2315-2326.

14. Pereira Neto AF. A representação dos usuários no conselhos de saúde: uma contribuição para o debate. Physis 2012; 22(2):441-462.

15. Silva LMS, Silva MRF, Lima LL, Fernandes MC, Oliveira NRN, Torres RAM. Análise da organizacão e funcionamento dos conselhos de saúde e a gestão participativa em Fortaleza-CE. Saúde Soc 2012; 21(Supl. 1):117-125

16. Cotta RMM, Cazal MM, Martins PC. Conselho municipal de saúde: (re)pensando a lacuna entre o formato institucional e o espaço de participação social. Cien Saude Colet 2010; 15(5):2431-2436.

7. Olson M. A lógica da ação coletiva: os benefícios públicos e uma teoria dos grupos sociais. São Paulo: EDUSP; 1999.

18. Farias Filho MC, Arruda Filho EJM. Planejamento da pesquisa científica. São Paulo: Atlas; 2013.

19. Cozby PC. Métodos de pesquisa em ciências do com portamento. São Paulo: Atlas; 2003.

20. Brasil. Ministério da Saúde (MS). Conselho Nacional de Saúde. Resolução no. 196 de 10 de outubro de 1996. Diretrizes e Normas Regulamentadoras de Pesquisas Envolvendo Seres Humanos. Diário Oficial da União 1996; 16 out.

21. Landerdhal MC, Unfer B, Braun K, Skupien JA. Resoluções do conselho de saúde: instrumento de controle social ou documento burocrático? Cien Saude Colet 2010; 15(5):2437-2445.

22. Brasil. Ministério da Saúde (MS). Resolução no 333 de 4 de novembro de 2003. Aprova as diretrizes para criação, reformulação, estruturação e funcionamento dos conselhos de saúde. Diário Oficial da União 2003; 4 dez.

Artigo apresentado em 18/03/2013

Aprovado em 23/05/2013

Versão final apresentada em 29/05/2013 
